\title{
Solid state excimer lasers
}

\author{
G. ZERZA, G. SLIWINSKI* and N. SCHWENTNER
}

Institut für Experimentalphysik, FU Berlin, Arnimallee 14, 14195 Berlin, Germany

${ }^{*}$ Polish Academy of Sciences, IF-FM, Fiszera 14, 80952 Gdansk, Poland

Doped rare gas crystals represent an interesting group of laser materials for which stimulated emission and lasing has been demonstrated in the UV and visible spectral range $11,2 /$. We report the results of an extensive investigation of $\mathrm{Ar}$ and $\mathrm{Ne}$ solids binary doped with $X_{e}$ and $F_{2}$ for laser applications /2,3/. The $X_{e} F_{\text {population is }}$ photochemically prepared with high quantum efficiency and excited state densities in the range of $1018 / \mathrm{cm}^{3}$ are senerated. The emission lines are slightly red shifted in comparison with the gas phase due to the dielectric interaction with the host. The XeF cross sections for stimulated emission of $10^{-18}-10^{-18} \mathrm{~cm}^{2}$ together with high inversion dengities result in large gain coefficients, e.g. for the $D \rightarrow X(286 \mathrm{~nm}), \mathrm{B} \rightarrow \mathrm{X}(411 \mathrm{~nm})$ and $\mathrm{C} \rightarrow \mathrm{A}(54.0 \mathrm{~nm})$ transitions. Values of 11,6 and $7 \mathrm{~cm}^{-1}$ are measured by rneans of various methods. Experiments with optimized cavities allow for extraction of low divergence beams ( $3 \mathrm{mrad}$ ) with quantum efficiencies up to $14 \%$. Inhomogeneously broadened spectral profiles and relaxation oscillations are observed in the $\mathrm{C} \rightarrow \mathrm{A}$ laser emission of $\mathrm{XeE}$ in solid Ar.

References:

11) N. Schwentner, V.A. Apkarian; Chem.Phys.Leit.154,413,(1989)

/2/ G. Zerza, G. Sliwinski and N. Schwentner; Appl.Phys.B55,331,(1992)

/3/ G. Zerza, G. Sliwinski and N. Schwentner; Appl.Phys.A.56,156,(1093) 